\title{
Bulls and Bears: \\ Lessons from some European Countries
}

\author{
Javier Gómez Biscarri (YE) \\ Fernando Pérez de Gracia * \\ IESE - University of Navarre \\ University of Navarre
}

\begin{abstract}
This paper analyzes the recent behavior of stock markets in four European countries. More specifically, we describe the bull and bear phases of those markets, comparing some of their more relevant features across countries and with those of the US. We also comment on the degree of concordance of stock market phases across countries. We find that cycles in European countries have become substantially more concordant in the recent years, a result that was to be expected given the increased integration of the European financial markets, but that the degree of concordance is not high.
\end{abstract}

JEL Classification: C41, G1

Keywords: stock market, bull, bear, concordance of cycles, European countries.

${ }^{*}$ School of Economics, University of Navarre, Campus Universitario, 31080 Pamplona, SPAIN, fgracia@unav.es. Javier Gómez Biscarri: IESE Business School and School of Economics, University of Navarre, Campus Universitario, 31080 Pamplona, SPAIN, jgbiscarri@iese.edu. 


\section{Introduction}

Bull and bear markets have been singled out as one of the main sources of medium to long term movements in the stock market over the past decades. Two explanations have been put forward for the existence of bull and bear markets. One view, held by Keynes (1936), Galbraith (1954) and Shiller (1989) among others, is that major bull and bear markets are purely due to irrational "animal spirit." An alternative view (DeLong, 1992; Siegel, 1998) states that, although prices deviate from fundamentals in the short run, that is, in periods of months or a few years, in the long run (decades or even generations), proportional differences between market prices and fundamentals are kept within bounds. Under this interpretation, the major bull and bear markets reflect large shifts in consensus perceptions of fundamentals and expectations of the future. ${ }^{1}$

As a consequence of the direct applicability of business cycle techniques, and of the increased general interest in the stock market, the behavior of bull and bear markets in stock prices has received considerable attention in the recent literature. Maheu and McCurdy (2000) identify bull and bear markets in stock returns using a Markov-switching model that incorporates duration dependence to capture non-linear structure in both the conditional mean and the conditional variance of stock returns. Lunde and Timmermann (2000), for example, analyze the presence of bull and bear market states in stock prices. Their definition of bull and bear is based on sequences of stopping times tracing local peaks and troughs in stock prices. Duration is examined through posterior mode estimates of the hazard function in bull and bear market. Pagan and Sossounov (2002) present a simple framework for analyzing bull and bear markets based on traditional business cycle analysis in the Bry-Boschan spirit. They use simulation methods in order to examine a number of data generating processes that are known to fit the data quite well -random walks, GARCH models and the switching regime model with duration dependence. Their main result is that a pure random walk with drift provides a good explanation of the characteristics of US bull and bear markets. Edwards et al. (2002) perform a similar analysis using emerging stock market data. They find that bull and bear cycles in emerging countries tend to have shorter duration and bigger amplitude than in developed countries. Their shape also shows significant departures from the triangular shape that would be implied by the most widely used statistical stock market return models and by the efficiency of the stock market.

However, the content of the papers cited above is mainly of a technical nature. Very few comments on the economic significance of the results are provided. In this paper we follow the analysis in Pagan and Sossounov (2002) to analyze the recent behavior of the stock market in four European countries but we take a diverging road, and place our emphasis on the economic significance of the results obtained. More specifically, we date and describe the bull and bear phases of the stock market of four European countries, comparing their characteristics during both phases and the degree of concordance of bullish periods

\footnotetext{
${ }^{1}$ The already famous debates between Profs. Robert Shiller and Jeremy Siegel are direct consequence of these two points of view: see for example Shiller (2000) and Siegel (1998).
} 
across countries in Europe. We try to put the results in context by analyzing what they imply in terms of economic and financial integration in Europe. Europe is a most notable example of increased integration. We believe that a thorough study of the degree to which European stock markets present cycles and of the joint characteristics of these cycles may yield interesting conclusions regarding financial fluctuations.

The paper is organized as follows. In Section 2 we explain the algorithm used to find the turning points between the phases and develop a battery of measures that characterize the behavior of stock prices during the bull/bear phases of the markets. In Section 3, we discuss the results of applying these measures to the bull and bear phases of the stock markets of four Eurozone countries: Spain, Italy, France and Germany. In Section 4 we deal with issues of concordance or synchronicity of the cycles across countries. Section 5 concludes.

\section{Dating Market Phases: Methodological Issues}

Our first concern in this paper is the identification of the phases of the different European stock markets. Once the phases have been identified, by locating the peaks and troughs of a stock index, we calculate some descriptive measures: Duration of the phases, average return during the phase, shape in terms of the deviation from a triangular approximation (see Figure 1), volatility within the phase and the proportion of big swings. This allows us to comment on the features of each stock market, and to compare their evolution with that of the US. We present in the next subsections the algorithm that locates the turning points and the measures used to characterize the cycle. Section 3 presents the results of applying the analysis to our emerging markets of interest.

\subsection{Locating Peaks and Troughs}

In order to determine the different phases of the stock market we modify the Bry-Boschan business cycle dating algorithm (Bry and Boschan, 1971) as operationalized by Watson (1994). In particular, we implement the censoring procedures suggested by Pagan and Sossounov (2002) and we do not locate the peaks and troughs on a moving average of the data, but rather on the original series of stock prices.

We first proceed to locate the turning points, peaks and troughs, of the stock index series, which determine the moment when the market changes from bullish to bearish and viceversa.

A peak/trough in the series of stock prices $P_{t}$ is defined if $p_{t}^{2}$ is the highest/lowest in a window of width $8 .^{3}$ That is, there is a peak at $t$ if

$$
\left[p_{t-8}, \ldots, p_{t-1}<p_{t}>p_{t+1}, \ldots, p_{t+8}\right]
$$

\footnotetext{
${ }^{2}$ Throughout the paper, $p_{t}$ denotes the natural $\log$ of the stock price, $\ln \left(P_{t}\right)$. It is clear that the peaks and troughs for both series have to be the same.

${ }^{3}$ The results may be slightly sensitive to the choice of the window width. We use eight months as suggested by Pagan and Sossounov (2002).
} 
and there is a trough at $t$ if

$$
\left[p_{t-8}, \ldots, p_{t-1}>p_{t}<p_{t+1}, \ldots, p_{t+8}\right]
$$

We include four censoring criteria to ensure that we do not identify spurious phases:

1) We eliminate turns within eight months of the beginning/end of the series.

2) Peaks or troughs next to the endpoints of the series are eliminated if they are lower/higher than the endpoints.

3) Complete cycles of less than 16 months of total duration are also eliminated.

4) Phases of less than four months are eliminated unless the fall/rise exceeds $20 \%$ (the traditional rule of thumb for identifying a stock market cumulative movement as bullish or bearish).

After every censoring operation, alternation is enforced so that a peak will always follow a trough and viceversa. Alternation is achieved by taking the highest (lowest) of two consecutive peaks (troughs).

\subsection{Describing Bull and Bear Phases}

In order to describe accurately the characteristics of each country's stock market cycles we are going to use six different measures of cyclical behavior. The first four directly relate to the shape of the phases of the cycle, while the other two focus on volatility within the cycle and on the existence of "big swings."

We need to use an indicator of whether the market is in a bull or bear phase to be able to compute the different measures. We define a dummy variable, $S_{t}$, which takes the value 1 if there is a bull market at time $t$, i.e. if $p_{t}-p_{t-1}=\Delta p_{t}$ belongs to the bull market. ${ }^{4}$ Given that $S_{t}=1$ if there is a bullish market at $t$, we first calculate two ancillary statistics: Total time spent in an expansion is $\sum_{t=1}^{T} S_{t}$ and total time spent in a contraction is $\sum_{t=1}^{T} B_{t}$, where $B_{t}=1-S_{t}$ is an indicator for bearish market. The Number of peaks (expansions) is NTP $=$ $\sum_{t=1}^{T} S_{t}\left(1-S_{t+1}\right)+1$ (NTP is short for "Number of Trough to Peak") and, of course, the Number of troughs (contractions) is $N P T=\sum_{t=1}^{T} B_{t}\left(1-B_{t+1}\right)+1$.

The six characteristics of the phases of the market that we study are:

1) Duration (D): average duration in months of expansions and contractions can be calculated as

$$
\widehat{D}_{b u l l}=\frac{1}{N T P} \sum_{t=1}^{T} S_{t}, \widehat{D}_{\text {bear }}=\frac{1}{N P T} \sum_{t=1}^{T} B_{t}
$$

\footnotetext{
${ }^{4}$ This differs from Pagan and Sossounov (2002) for they use $S_{t}=1$ if $p_{t+1}-p_{t}=\Delta p_{t}$ is bullish. There is therefore a slight difference with respect to the above paper in some of the formulas that we present. Note that the bull market corresponds to the periods between a trough and a peak of the series, and the bear market to the periods between a peak and a trough.
} 
2) Amplitude (A): average amplitudes of the phases, in percentage, are

$$
\widehat{A}_{\text {bull }}=\frac{1}{N T P} \sum_{t=1}^{T} S_{t} \Delta p_{t}, \widehat{A}_{\text {bear }}=\frac{1}{N P T} \sum_{t=1}^{T} B_{t} \Delta p_{t}
$$

To get the cumulated change over any expansion we define $Z_{t}^{\text {bull }}=S_{t} Z_{t-1}+$ $S_{t} \Delta p_{t}, Z_{0}=0 . Z_{t}$ contains the running sum of returns $\Delta p_{t}$ for bullish markets (similar definition with $B_{t}$ for bearish markets) with the sum reset to zero whenever $S_{t}=0$.

3) Cumulated Change: total cumulated change (TC) over expansions and contractions, in percentage, is

$$
\widehat{T C}_{\text {bull }}=\sum_{t=1}^{T} Z_{t}^{\text {bull }}=\sum_{t=1}^{T} \sum_{j=1}^{t} S_{j} \Delta p_{j}, \widehat{T C}_{\text {bear }}=\sum_{t=1}^{T} Z_{t}^{\text {bear }}=\sum_{t=1}^{T} \sum_{j=1}^{t} B_{j} \Delta p_{j}
$$

and the average cumulated change $(C)$ for both phases of the cycle is

$$
\begin{aligned}
& \widehat{C}_{\text {bull }}=\frac{1}{N T P} \sum_{t=1}^{T} Z_{t}^{\text {bull }}=\frac{1}{N T P} \sum_{t=1}^{T} \sum_{j=1}^{t} S_{j} \Delta p_{j} \\
& \widehat{C}_{\text {bear }}=\frac{1}{N P T} \sum_{t=1}^{T} Z_{t}^{\text {bear }}=\frac{1}{N P T} \sum_{t=1}^{T} \sum_{j=1}^{t} B_{j} \Delta p_{j}
\end{aligned}
$$

This measure can be interpreted as a discrete time approximation to the integral below (above) the bull (bear) market. It allows us to see how much the behavior of the phase differs from a purely triangular cycle, which is the behavior prices would follow if a random walk model was the true data generating process. We use this measure to calculate an excess index that will allow us to perform significance tests on possible departures from the random walk behavior.

4) Excess Index (EX): an index that measures the excess with respect to a triangular approximation can be calculated as

$$
\widehat{E X}=\frac{(\widehat{C}-0.5 \widehat{A}-0.5 \widehat{A} \cdot \widehat{D})}{\widehat{D}}
$$

for both expansions and contractions.

5) Volatility $(V)$ : We use a measure of variability within each phase, that is a crude way of seeing how volatile the price is in each of the two phases. Instead of the squared changes in prices $\Delta p_{t}^{2}$ we use the absolute value $\left|\Delta p_{t}\right|$, to make sure that the index is less sensitive to unusually big changes which frequently occur in stock markets. Our measure of variability is therefore

$$
V^{\text {bull }}=\frac{1}{N T P} \sum_{t=1}^{T} S_{t}\left|\Delta p_{t}\right|, V^{\text {bear }}=\frac{1}{N P T} \sum_{t=1}^{T} B_{t}\left|\Delta p_{t}\right|
$$


6) Proportion of big expansions $\left(B^{+}\right)$and contractions $\left(B^{-}\right)$: The proportion of expansions and contractions that are bigger than 0.2 or smaller than -0.2 in percentage return is:

$$
\begin{aligned}
B^{+} & =\frac{1}{N T P} \sum_{t=1}^{T} I\left[S_{t}\left(1-S_{t+1}\right) Z_{t}^{\text {bull }}>0.2\right] \\
B^{-} & =\frac{1}{N T P} \sum_{t=1}^{T} I\left[B_{t}\left(1-B_{t+1}\right) Z_{t}^{\text {bear }}<-0.2\right]
\end{aligned}
$$

The summation is taking, of the running sum of each expansion $\left(Z_{t}^{\text {bull }}\right)$ or contraction $\left(Z_{t}^{\text {bear }}\right)$ only the value at the peak/trough. This is equivalent to the amplitude of the phase. The indicator function $I[a]$ is therefore just counting the number of phases with amplitude bigger than 0.2 or smaller than -0.2 .

One of the advantages of our approach is that it allows us to compare the actual stock market cycle to a benchmark characterized by a random walk. As pointed out above, if the true data generating process is indeed a random walk, the actual cyclical behavior would follow a triangular pattern. If, however, actual cycles depart from those coming from a random walk, the cyclical pattern could take a number of alternative forms. Figure 1 depicts the four possible shapes of the market behavior depending on whether the market is bullish or bearish and the excess index is positive or negative. One can think of these shapes as telling us when the phase "accelerates," that is, where in the phase, either at the beginning or at the end, the biggest returns are located. A positive excess measure in a bullish market will then say that the first periods of the phase are those of higher (accelerated) returns whereas a positive excess measure in a bearish market would say that it is at the end of the phase that the bigger negative returns happen. The opposite would be true of negative excess measures.

\section{[Insert Figure 1]}

Given that we can estimate the parameters of any statistical model for all our candidate countries, we can use those parameters to simulate the process under that data generating process as null hypothesis. Thus, we can construct a distribution of the $E X$ index under that null hypothesis and perform a formal test. This index therefore would allow us to give statistical evidence for or against the data generating processes that is not captured with the usual significance tests or out-of-sample forecasting exercises. ${ }^{5}$

\footnotetext{
${ }^{5}$ In theory, all six measures could be used in this way to test some statistical model. However, it turns out that most statistical models can replicate fairly well all measures except for the excess index. This index, therefore, turns out to be the one that can most successfully be used for a formal testing of whether a statistical model is consistent with observed market behavior.
} 


\section{Bull and Bear Phases in some European Coun- tries}

We investigate market phases in four European countries that have long time series available of stock market data: Spain, France, Italy and Germany. In order to investigate the characteristics of the bull and bear phases of these markets we use the longest series of each country's stock market index available to us. These correspond first to a monthly data set of a Spanish stock index running from 1/1941 to 11/1999 and obtained from the Research Department of the Madrid Stock Exchange. ${ }^{6}$ Additionally, we use monthly data from the IFS database of the IMF for France, Italy and Germany. In the case of France and Italy we have available data from $1 / 1957$ to $11 / 1999$. In the case of Germany, data are available from $1 / 1970$ to $11 / 1999$.

Table 1 presents the main characteristics of the bull and bear markets of the four European countries that we analyze in this paper. For the sake of comparison, we present in the last column of Table 1 bull and bear market statistics for the US stock market, during the period 1/1945 - 5/1997, estimated by Pagan and Sossounov (2002).

\section{[Insert Table 1]}

Also, we present in Figures 2 to 5 the evolution of the stock indexes for each of the countries, where the bullish periods have been shaded to facilitate visual inspection.

[Insert Figures 2 to 5]

\subsection{Basic Results}

The main stylized facts about the bull/bear markets in our European countries can be summarized as follows.

First, it is clear that bull phases, with an average duration of around 30 months, tend to be longer than bear phases, that usually last for around 14 months. Germany and the US, that we could argue are the countries with the most developed stock markets, present both shorter bullish (25 months) and bearish (13 months) phases. Italy is an exception, since the average duration in that country is 19.5 months for bull markets and 20 months for bear markets. These figures contrast markedly, for instance, with those for emerging markets, where bull markets are on average shorter (around 20.3 months) but bear markets present a similar duration (16.6 months). ${ }^{7}$

\footnotetext{
${ }^{6}$ The cooperation of the Madrid Stock Exchange in providing the long time series of data is gratefully acknowledged.

${ }^{7}$ Brazil is also an exception in the group of emerging markets. The Brazilian stock market presents an average duration of 17.8 months for bull phases and 19.9 months for bear phases. On emerging countries see Edwards et al. (2002).
} 
Average amplitude for bull markets is greatest in the case of Spain, where the average capital gain over the bullish phase is of around $78 \%$. The largest bear markets are those of Italy, where the average capital loss over the phase is of $40 \%$. In the emerging countries, the amplitude of both bull and bear phases is much bigger than in the case of European countries or the US. ${ }^{8}$ One interesting finding is that both Germany and the US present bullish and bearish phases with smaller amplitude than the rest of the countries. Not only, therefore, are their phases shorter, but the average return/loss over the phase is also smaller.

The results on the cumulated change $(C)$ are needed to calculate the excess index, so we do not discuss them since their interpretation is not immediate and they do not offer much insight. The excess index, on the other hand, gives some relevant information. It reflects how the actual path of the bullish or bearish market deviates from a triangle approximation. A pure random walk with no drift would produce triangular cycles, with excess measures not significantly different from zero in either bull or bear phases. Random walks with drift tend to produce bullish phases with positive excess measure and bearish phases with negative excess measure, and this effect is accentuated when autocorrelation or conditional heteroskedasticity are allowed.

The Spanish stock market exhibits expansions that deviate quite substantially from a triangle approximation. ${ }^{9}$ This is not true of the rest of the countries, where the value of the excess index is four to five times smaller than that of Spain. In the case of bear markets, Italy is the only country that presents a high value of the $E X$ index, though negative. Note that these two correspond to the countries with the longest bull and bear markets respectively. Thus, the evidence would seem to favor the fact already found in Pagan and Sossounov (2002): Stock market phases do not look triangular, as it would be implied by a simple random walk, but instead the first periods of the bullish phase seem to be ones of accelerated growth (positive excess measure) just as it is the first periods of the bearish phase that tend to display accelerated losses (thus yielding a negative excess measure). However, we show in the next subsection that the values of the $E X$ index for all four European countries are perfectly coherent with the behavior of a simple random walk with drift and of a simple random walk with drift and some positive autocorrelation in the returns. Thus, in the case of European markets the $E X$ index cannot discriminate between a model with some predictability and an unpredictable model: Both are coherent with the empirical features of the European data. Notice that this finding qualifies the statement in Pagan and Sossounov (2002): It is certainly true that the more the market path deviates from a triangle approximation, the more it departs from a random walk type of behavior, but it is not the case that all deviations reflect some kind of predictability. A random walk with drift but no autocorrela-

\footnotetext{
${ }^{8}$ Emerging stock market seem to be offering a significant risk premium during expansions, that compensates for the big losses during contractions and for the substantially higher volatility over the cycle.

${ }^{9}$ It is possible that the results in the case of Spain are too strongly influenced by the second half of the sample period, since visual inspection of Figure 1 reveals that bull phases up to the 1970's had negative excess from the triangle approximation.
} 
tion (and therefore, unpredictable beyond the regular market return) generates cycles with positive excess measure. Therefore, if we want to test whether there is some predictability in the market we need to see what values of the $E X$ index are consistent with an unpredictable random walk and what values are not, but the benchmark value will not be zero if there is a drift in the market. This we do in the next subsection.

Our measure of volatility within the phase is in all cases greater in the bull market than in the bear market, a fact that should not be surprising given the overall upward drift of the four markets. If we look across countries, we find that France and Spain suffer significantly higher volatility within the bull phase while Italy again presents the most volatile bear phase. It seems that longer duration and amplitude of the phase are related to increased volatility.

Finally, the proportion of "big swings" reflects that the Spanish and French markets have a similar number of expansions and contractions bigger than 0.2 and smaller than -0.2. Italy tends to have fewer big bearish swings. We have seen, however, that the average amplitude of Italian bear phases was much bigger than that of the rest of the countries. Thus, the conclusion follows, and can be checked in Figure 4, that Italy presents a few big bearish phases while also presenting numerous other phases longer than those of the other three countries, but not necessarily big in amplitude. Germany, too, presents a high proportion of "big bullish swings" and fewer bearish swings.

For the sake of comparison, we have presented in the last column of Table 1 bull and bear market statistics for the US stock market, during the period 1/1945 - 5/1997, estimated by Pagan and Sossounov (2002). The correspondence between our results for some European markets and the results proposed by Pagan and Sossounov is high. Comparison between US and European markets shows that the German market is the most similar to the American market in duration, amplitude and the proportion of "big swings" during expansions and contractions. The rest of the European countries all show some clear differences with respect to the behavior of the US stock market, these differences being the most noticeable in the case of Italy.

As mentioned above, the duration, amplitude and volatility of the market cycles can be well accounted for by classical models of stock market behavior (see Gómez Biscarri and Pérez de Gracia., 2002). However, the excess index provides us with a feature of the cycle that is not easily captured by statistical models of returns. In fact, the behavior of most actual stock markets seems to differ from what would be implied by a statistical model in a specific manner: Big returns (in absolute value) tend to be concentrated either at the beginning or at the end of the phase, but they are usually not evenly distributed, as would be implied by most statistical models. In the next subsection we perform a simulation exercise that tries to find the values of the $E X$ index that are consistent with statistical models of market returns and to test whether the observed values of the index for the European countries can therefore be generated by the market models. 


\subsection{Actual Cycles vs the Random Walk Benchmark: A Simulation Exercise}

In order to gain a deeper understanding of whether stock market phases depart from what the random walk would imply, we perform a simulation experiment.

First we fit three statistical models of returns to all four of our markets. These three models differ with respect to the behavior of the volatility of the error term. The three models estimated are:

a) A random walk with drift and $\operatorname{AR}(1)$ increments

$$
\Delta p_{t}=\mu+\rho \Delta p_{t-1}+\sigma \varepsilon_{t}, \varepsilon_{t} \rightarrow \operatorname{nid}(0,1)
$$

b) A random walk with drift and $\operatorname{AR}(1) / \operatorname{GARCH}(1,1)$ effects

$$
\begin{aligned}
\Delta p_{t} & =\mu+\rho \Delta p_{t-1}+u_{t}, u_{t} \rightarrow \operatorname{nid}\left(0, h_{t}\right) \\
h_{t} & =\alpha+\beta u_{t-1}^{2}+\gamma h_{t-1}
\end{aligned}
$$

c) A random walk with drift and $\mathrm{AR}(1) / \mathrm{EGARCH}(1,1)$ effects

$$
\begin{aligned}
\Delta p_{t} & =\mu+\rho \Delta p_{t-1}+\sigma_{t} \varepsilon_{t}, \varepsilon_{t} \rightarrow \operatorname{nid}(0,1) \\
\ln \sigma_{t}^{2} & =h_{t}=\phi_{0}+\phi_{1} \varepsilon_{t-1}+\phi_{2}\left|\varepsilon_{t-1}\right|+\phi_{3} \ln \sigma_{t-1}^{2}
\end{aligned}
$$

All three models are very standard and were chosen because they are the ones that best replicate the empirical features of the cycles, besides being simple to estimate (Pagan and Sossounov, 2002). Notice that they all include the possibility of returns being autocorrelated.

Second, we simulated 1,000 series of returns (of length equal to the number of observations available in each country's stock market series) for all four countries, given the estimated parameters for the above three models. We calculated the excess measures for each of the 1,000 simulated series and constructed the simulated distribution of the excess measure under each of the three models. From these simulated distributions we find the $2.5 \%$ and $97.5 \%$ critical values.

Third, we compared the values of the empirical excess measures estimated for the original series of the four countries (rows 4 and 10 in Table 1), with the critical values that result from simulating the stock market with the estimated parameters of the three models. ${ }^{10}$

The conclusion that comes out from this experiment is that in all cases the values of the $E X$ index that we find in real markets can come from any of the three statistical models considered. All three models can replicate quite closely most of the features of the bull and bear phases of the different countries (duration, amplitude, proportion of big phases and volatility are very well accounted for) including the $E X$ index. This is relevant especially if we consider that in the case of emerging markets this result did not hold at all (See Edwards et al, 2001). For most of the emerging markets analyzed by the authors, they could find no statistical model that would replicate the shape of the cycle, a result

\footnotetext{
${ }^{10}$ The simulated critical values are available upon request. We omit them from the exposition in order to simplify the flow of the text.
} 
which pointed at further predictability in those markets than that coming purely from a mild autocorrelation in market returns. In the case of Europe, where the stock markets are more developed and efficient, this result does not appear and we are not able to find departures from a simple random walk-AR(1) behavior in market returns.

These results are important, for they could have pointed at a feature of stock market predictability that cannot easily be accommodated by statistical models and that is not apparent from a casual examination of the series. It is true, though, that Figures 2 to 7 do give some evidence of this result, but it is always difficult to disentangle the part of the departure from the triangle path that could be due to the drift of the random walk or to variance effects. More complicated statistical models, such as a stochastic volatility model or a regime switching model "a la Hamilton" also fail to replicate this effect, plus they do a poor job in replicating some of the other features (Gómez Biscarri and Pérez de Gracia, 2002, for a more complete analysis of the case of Spain that includes these models). In the case of a two regime switching model (Hamilton, 1989), for example, the two regimes are indeed identified with the bull and bear phases, but no distinction is made between the behavior of the series during the first and last months of each of the phases, a feature that, as we have seen, appears in the data analyzed. ${ }^{11}$

\section{Bull and Bear Phases and Integration of Eu- ropean Stock Markets}

We turn now to a different analysis, where we are going to look at the joint evolution of the four European stock markets we have been analyzing so far. We are interested in seeing whether European markets tend to move in the same direction, in opposite directions or whether their movements are not at all coordinated. The issue of financial integration in Europe comes obviously to mind. Ever since the EU removed in 1990 most barriers to capital flows within Europe, we should have witnessed an increase in flows among countries, and therefore enhanced integration of the domestic stock markets. Increased capital flows among European countries would lead to increased stock market concordance, either positive or negative. The efficient market hypothesis would imply that if shocks to the countries are independent, their stock markets should also behave independently. If the shocks are highly correlated, the direction of this correlation would determine the comovement of the stock markets. Since financial shocks seem to be more and more concordant within the EU, both for the effect of the EMS and for the increased economic integration, one would expect that European stock markets would be more and more concordant, that is, they would tend to be more frequently in a similar stage or phase of the cycle.

\footnotetext{
${ }^{11}$ Maybe a four regime model would capture this feature, but this complicates immensely the estimation process.
} 
We present in the next subsections the concordance index, which help us analyze the degree to which European stock markets behave similarly, a set of rolling concordance indexes which show whether this concordance has been evolving during the recent years and the peaks and troughs of the bull and bear cycles phases.

\subsection{Concordance of Cycle Phases}

In order to analyze the comovements of the stock market data in our four countries, we use a concordance index of the bullish and bearish phases that compares how aligned or concordant the cycle phases in two different countries are. This concordance index, used for example in Harding and Pagan (2002), allows us to infer the extent to which the stock market phases of these countries are correlated.

The concordance index (CI) is calculated, for countries $i$ and $j$, as

$$
C I_{i j}=\frac{1}{T} \sum_{t=1}^{T}\left[S_{t}^{i} \cdot S_{t}^{j}+B_{t}^{i} \cdot B_{t}^{j}\right]=\frac{1}{T} \sum_{t=1}^{T}\left[S_{t}^{i} \cdot S_{t}^{j}+\left(1-S_{t}^{i}\right) \cdot\left(1-S_{t}^{j}\right)\right]
$$

which of course is just calculating the number of the $T$ periods for which the two countries are in the same phase, either bull or bear, and averaging out over the $T$ periods.

There are other possible measures of concordance of the cycles. In fact, one could think that pure cross-correlations of returns are simpler to calculate and would work better. There are three main problems with cross-correlations, however. First, unconditional cross-correlations of returns are subject to sectorial composition of the stock indexes and do not account for movements in fundamentals (see Wolf, 1996). ${ }^{12}$ Second, the use of simple cross-correlations would be affected by the existence, not infrequent in the case of stock market movements, of outliers. By identifying the phases with a binary variable, we avoid the artificial increase in cross-correlation that would result from extremely big returns. In fact, the $C I$ can be interpreted as a pseudo-correlation of two binary variables. Finally, the behavior of volatility or of higher moments of the returns would affect the cross-correlation measure of the concordance of cycles, whereas the $C I$ is invariant to the underlying distribution of returns once the phases have been identified. For these three reasons we believe that this $C I$ provides an intuitive measure of the concordance of the cycle phases, and, given the objective of our study, a more correct one.

If the phases in two countries were perfectly aligned, we would find a $C I$ of 1 , and if they were perfectly misaligned we would find a $C I$ of 0 . However,

\footnotetext{
12 There are still some additional reasons for not using a return correlation type of measure. One has to keep in mind that we are not interested in forecasting returns or in the short term movements of the market. Our main concern is to calculate the concordance of phases in the stock markets, and these phases correspond to medium to long run movements. Using cross-correlations of returns would put too much emphasis on short term movements. The methodology used to construct the phase indicators guarantees that we focus on the medium term trends of the stock market.
} 
given that random walks with drift generate cycles with longer and bigger bullish phases than bearish phases, it is clear that even if the shocks to the random walks are perfectly independent, the concordance index will be slightly bigger than 0.5 , the value we would obtain if the phases were purely random draws from a 50:50 Bernoulli distribution. Therefore, critical values for testing the significance of the indexes have to be calculated case by case in a manner similar to those for the excess measures. We simulated independent random walks with the same drift, return autocorrelation and variance as the country series. Then we calculated the simulated distribution of all bilateral concordance indexes and found the $2.5 \%$ and $97.5 \%$ critical values. These critical values are then compared to those obtained from the series. When the empirical index is outside of the confidence interval, one can reject the hypothesis that the two series have phases that come from independent processes, which gives evidence of significant concordance of stock market behavior in those two countries.

In Table 2 we present the bilateral concordance index between the different stock indexes over the same period.

[Insert Table 2]

Values of the index are all bigger than 0.5 , but they are in general not big enough to justify the claim that the markets are subject to correlated phases. The results show that all four European countries show a similar degree of concordance in their cycles. However, the values of the bilateral indexes are not above the critical level for three of the pairs. Only two of the indexes are above the critical level of positive concordance, namely those of Italy and France and of Germany and Italy. One can see, though, that the value of the index for the three non-significant pairs (Spain vs. the other three markets) is very close in magnitude to the two others, though slightly below the critical value. One is led to think therefore that cycles in the Spanish market are slightly less synchronized with the rest of Europe than they are among other European countries.

The value of this index, however, may be missing historically important facts. For instance,during the first years of the sample there were barriers to capital mobility across European countries, and there was little financial integration in Europe. After 1990 barriers to capital mobility were abolished among EU members, thus facilitating increased capital flows among the member countries. Consequently, an index that only looks at the long-run concordance of the phases is probably missing the time evolution of capital market integration. In the next subsection we analyze whether the time evolution of the $C I$ supports the story of increased market concordance.

\subsection{Rolling Concordance Index of Cycle Phases}

In this subsection we focus on time evolution of stock market concordance in Europe. For that purpose, we calculate a rolling concordance index $(R C I)$ 
for our four European countries. We present in Figures 6-7 two sets of rolling concordance indexes, calculated using a rolling window of width 200. That is, we calculate the $R C I$ for countries $\mathrm{i}$ and $\mathrm{j}$ as

$$
R C I_{i j}^{\tau}=\frac{1}{200} \sum_{t=\tau-199}^{\tau}\left[S_{t}^{i} \cdot S_{t}^{j}+B_{t}^{i} \cdot B_{t}^{j}\right]
$$

and we then plot $R C I_{i j}^{200}$ to $R C I_{i j}^{T}$ in the graphs. These rolling coincident indexes give us an idea of how the degree of concordance has been changing over time. Here again, formal testing of whether these indices show significant concordance have to be constructed from simulated critical values. We simulated critical values for the rolling coincident indexes, by generating series with the estimated drift and return autocorrelation of each of the four countries and calculating the rolling coincident indexes and the $95 \%$ confidence bands by taking the $2.5 \%$ and the $97.5 \%$ percentiles of the simulated values in each period. Given that the drifts are similar, the confidence bands turn out to be also similar, and thus we only include in the graphs one set of confidence bands, though, strictly speaking, there should be one set of bands per pair of countries. Notice that the confidence bands look like straight lines: They are not exactly straight, but they are very flat over time. We chose the most conservative set of bands, that is the smaller and bigger of the critical values of all the simulated bands. The confidence bands we use fluctuate, though it cannot be appreciated, around $[0.36,0.715]$.

In terms of significance levels, the results agree with those of the point estimates in Table 2 although they add a couple of qualifications. The $R C I$ of Germany and Italy is above significance levels since march 1992, but not during the first periods of the sample. It is noticeable the upward time trend that the index follows: more and more the stock market phases of these two countries seem to coincide over time. The $R C I$ between Italy and France is above significance levels from 1990 on, but again it is not significant during the first years of the sample (see Figures 6 and 7 ). The $R C I$ between Spain and Germany qualifies the result of the $C I$ that did not give evidence of significance concordance of stock market phases between these countries. The RCI shows a clear increasing time trend, and its value ends up being well outside the confidence interval at the end of the sample.

The case of Spain and France may be the most puzzling. There are instances where the index is above the interval band (in 1986-1987, 1988-1991 and 1993). It seems that during periods of stock market crisis the behavior of the French market tends to be more concordant with that of its close neighbor Spain, which is something one would expect, but it is indeed puzzling that the result does not hold for periods of market tranquility. The French and the Spanish economy seem to evolve in parallel manner, but this does not reflect on the stock market behavior. Another puzzling result is that the French stock market seems to bear no relationship with the German stock market: the value of the index is very low, well within nonsignificance levels despite a slight upward trend at the end of the sample, 
The most interesting conclusion comes therefore from looking at the upward trend of the $R C I^{\prime} s$ of Italy-Germany, Spain-Germany and France-Italy (and more mildly so, France-Germany). Given the increased degree of economic integration of European countries, and the increased liberalization of movements of capital across these markets, it is not unreasonable to think of European countries as becoming more an more an asset class, in the sense that investing in any two of those countries would be perceived as a substitute. Thus, money would flow in and out of the European stock markets simultaneously and the national markets should show concordant phases. Of course, this implies that the countries have also become concordant in terms of the shocks to the economy, a condition necessary for projects like the single currency to work.

Additionally, it is worth mentioning again the results regarding the behavior of the French stock market, which seems to be concordant with Spain and Italy only during periods of high instability, contrary to what one could expect.

\subsection{Peaks and Troughs in Bull and Bear Phases}

In order to further analyze stock market comovements, we use Figures 2-5 and the dates of the identified peaks/troughs in Table 3. In Figures 2-5 we have shaded the bullish phases for each of the four European markets.

\section{[Insert Tables 3a-3b]}

Tables $3 \mathrm{a}$ and $3 \mathrm{~b}$ present the dates of the peaks and troughs identified for all the countries in our analysis. For the sake of comparison, we present in the last column of the tables the peaks and troughs identified for the US by Pagan and Sossounov (2002). We have tried to align the dates of the peaks and troughs that happen at similar months, so that we can more easily check if turning points coincide in time or not. Bullish phases therefore range from the month after the trough date until (and including) the month of the peak. Bearish phases go from the month after the peak until (and including) the month of the trough. There is one clear fact from the analysis of the peaks and troughs for the different countries, complementary to what we find in Figures 6-7 and in Figures 2-5. Before 1987, the peaks and troughs of our four European countries seem to be totally different, and very different also from those identified for the US. There does not seem to be any significant alignment of the turning points in the four stock markets, all of which seem to follow widely differing patterns. For instance, Spanish bullish phases in the 80's correspond to 1979:11-1981:08, 1982:09-1987:08; 1987:11-1989:11, whereas those of Germany are 1980:03-1981:07, 1981:12-1986:04, 1988:01-1990:07. Peaks and troughs are not aligned (see Table $3 \mathrm{~b}$ ) until the end of 1987, when three of our countries, besides the US, present a trough almost simultaneously at the time of the crash of the US stock market. The pattern again breaks down until the end of 1990, where four countries (and the US) present a trough coinciding with the last worldwide recession. After 1990, however, the markets seem to enter 
into phase, and we see that the peaks and troughs, and therefore the bullish and bearish phases, begin to coincide in time. For instance, troughs coincide in 1992:09 (the beginning of the European Monetary System crisis), early 1995 (after the European Monetary crisis), and the final one in late 1998 (after the Russian crisis).

As we see, several of the financial crises seemed to contribute to the more intense comovements in the European markets. The way the markets react to financial crisis, and the increasing synchronicity of their stock market phases after 1990 hints again at the fact that European stocks markets, because of their growing financial integration and the increased real concordance in their economies, were considered a single alternative investment opportunity, and money flowed in and out of them simultaneously.

\section{Conclusions}

This paper studies the characteristics of bull and bear markets for some European countries. In order to detect cyclical patterns in stock prices movements we use the Bry-Boschan dating algorithm, as modified by Pagan and Sossounov (2002). We identify some characteristics of the bull and bear phases in the stock price cycles, such as average duration, average amplitude, excess from a triangle approximation, volatility within the phase and proportion of "big swings." These measures help us compare the cyclical behavior of the different stock markets. We find that cycles in Spain, France, Germany and Italy are generally similar, and they tend to have longer durations and smaller amplitudes than those of less developed countries. The shape of their cycles does not depart significantly from that implied by statistical models of unpredictable (or mildly predictable) returns. Additionally, we calculate concordance indexes for the countries in the sample, trying to detect whether the countries tend to be in the same phase of the stock market cycle or not. Our analysis gives evidence for significant coincidence between the group formed by Germany-Italy and Italy-France, but most importantly, it shows that European stock markets have tended to become more concordant over time, a fact that we attribute to enhanced real symmetry of their economies, and to the increased integration of European national financial markets in the 1990's. Further research in the area of stock market behavior and concordance or synchronicity of stock market cycles in Europe is definitely warranted, especially in the threshold of a possible enlargement of the Eurozone and of the EU, if negotiations with accession countries follow their due course.

\section{References}

Bry, G. and C. Boschan (1971): Cyclical Analysis of Time Series: Selected Procedures and Computer Programs. New York, NBER.

Edwards, S., J. Gómez Biscarri and F. Pérez de Gracia (2002): "Bull and Bear Cycles in Emerging Countries," University of Navarre, mimeo. 

Press.

Galbraith, J.K. (1954): The Great Crash. Cambridge, Mass.: Riverside

Gómez Biscarri, J. and F. Pérez de Gracia (2002): "Identifying and Replicating Bull and Bear Phases in the Spanish Stock Market," University of Navarre, mimeo.

Harding, D. and A.R. Pagan (2002): "Dissecting the Cycle: A Methodological Investigation," Journal of Monetary Economics (forthcoming).

Keynes, J.M. (1936): The General Theory of Employment, Interest and Money. Reprinted in The collected writings of John Maynard Keynes, vol.7, London: Macmillan, 1973.

Lunde, A. and A.G. Timmermann (2000): "Duration Dependence in Stock Prices: An Analysis of Bull and Bear Markets," UCSD, mimeo.

Maheu, J.M. and T.H. McCurdy (2000): "Identifying Bull and Bear Markets in Stock Returns," Journal of Business and Economic Statistics 18, pp. 100112.

Pagan, A.R. and K.A. Sossounov (2002): "A Simple Framework for Analyzing Bull and Bear Markets," Journal of Applied Econometrics, forthcoming.

Shiller, R.J. (1989): Market Volatility. Cambridge, Mass.:MIT Press.

Shiller, R.J. (2000): Irrational Exuberance. New York: Broadway Books.

Siegel, J.J. (1998): Stocks for the Long Run. New York: McGraw-Hill.

Watson, M.W. (1994): "Business-Cycle Durations and Postwar Stabilization of the U.S. Economy," American Economic Review 84, pp. 24-46.

Wolf, H. (1996): "Comovements among Emerging Equity Markets," in Glick (ed.) Managing Capital Flows and Exchange Rates. Perspectives from the Pacific Basin. Cambridge: Cambridge University Press. 
Table 1. Characteristics of the bull and bear markets for the specific countries

\begin{tabular}{lrrrrr}
\hline \multicolumn{1}{c}{$S P A I N$} & $F R A N C E$ & $I T A L Y$ & GERMANY & \multicolumn{1}{c}{$U S^{*}$} \\
\hline$N$ & 707 & 515 & 515 & 359 & \\
\hline Bull & $1 / 41-11 / 99$ & $1 / 57-11 / 98$ & $1 / 57-1 / 98$ & $1 / 70-11 / 99$ & $1 / 45-5 / 97$ \\
$D$ & 31.53333 & 32.27 & 19.462 & 25.33 & 26 \\
$A$ & 0.7956 & 0.6514 & 0.6557 & 0.495 & 0.46 \\
$C$ & 17.209 & 11.982 & 7.195 & 7.562 & 7.70 \\
$E X$ & 0.1353 & 0.0355 & 0.025 & 0.0412 & 0.03 \\
$V$ & 1.173 & 1.511 & 0.9059 & 0.8635 & -- \\
$B^{+}$ & 0.8 & 0.818 & 0.769 & 0.778 & 0.93 \\
\hline Bear & & & & & \\
$D$ & 15.5333 & 14.45 & 20 & 13 & 12 \\
$A$ & -0.3075 & -0.3358 & -0.4052 & -0.252 & -0.24 \\
$C$ & -3.065 & -2.453 & -6.539 & -2.222 & -1.60 \\
$E X$ & -0.0337 & 0.0098 & -0.113 & -0.0352 & 0.016 \\
$V$ & 0.6168 & 0.645 & 0.852 & 0.54 & -- \\
$B^{-}$ & 0.8 & 0.818 & 0.615 & 0.5 & 0.40 \\
\hline$N$
\end{tabular}

$\mathrm{N}$ : number of observations

$\mathrm{T}$ : period

D: average duration

A: average amplitude

$\mathrm{C}$ : average cumulative movement

EX: excess from a triangle approximation

$\mathrm{V}$ : volatility within the phase

B: proportion of "big swings"

* Pagan and Sossounov (2002) estimates on bull and bear markets in US stock price. 


\section{Table 2. Concordance Index (CI)}

The ${ }^{*}$ indicates that the value is outside of the $5 \%$ confidence interval. The confidence intervals have been calculated from simulated series using a Random Walk with Drift and AR(1) increments,

$$
\Delta p_{t}=\mu+\rho \Delta p_{t-1}+\sigma \varepsilon_{t}, \varepsilon_{t} \rightarrow \operatorname{nid}(0,1)
$$

that represents the best fitting model to the bull and bear cycles characteristics. Parameters of this model were estimated for all four countries, and then 10,000 series of length 302 were simulated and the CI calculated. The $2.5 \%$ and $97.5 \%$ quantiles of the simulated distribution of the CI are used as critical values. The specific values are available upon request. The critical values should be in the $0.35-0.66$ range, which is the common range for the rest of the series.

\begin{tabular}{rrrrrr}
\hline$C I$ & $S P A I N$ & $F R A N C E$ & $I T A L Y$ & GERMANY & $U S^{*}$ \\
\hline$S P A I N$ & 1 & 0.629 & 0.614 & 0.652 & 0.628 \\
$F R A N C E$ & & 1 & $0.643^{*}$ & 0.649 & 0.714 \\
$I T A L Y$ & & & 1 & $0.747^{*}$ & 0.631 \\
GERMANY & & & & 1 & 0.610 \\
\hline
\end{tabular}

* Pagan and Sossounov (2002) estimates on bull and bear markets in US stock price. Since we do not have the original data we cannot compute critical values and perform significance tests on these CI's. 
Table 3a. Dates of the Peaks and Troughs in the 1940-1969 period

\begin{tabular}{lcccc}
\hline & SPAIN & FRANCE & ITALY & US \\
\hline Peak & $1942: 09$ & N/A & N/A & \\
Trough & $1944: 01$ & N/A & N/A & \\
Peak & $1947: 03$ & N/A & N/A & $1946: 05$ \\
Trough & $1949: 06$ & N/A & N/A & $1948: 02$ \\
Peak & $1951: 02$ & N/A & N/A & $1952: 12$ \\
Trough & $1953: 04$ & N/A & N/A & $1953: 08$ \\
Peak & $1957: 01$ & & $1957: 09$ & $1956: 07$ \\
Trough & $1958: 06$ & & $1958: 06$ & $1957: 12$ \\
Peak & $1959: 01$ & & & $1959: 07$ \\
Trough & $1959: 11$ & & & $1960: 10$ \\
Peak & $1963: 02$ & $1962: 04$ & $1960: 09$ & $1961: 12$ \\
Trough & $1964: 05$ & $1964: 06$ & $1965: 01$ & $1962: 06$ \\
Peak & & $1966: 01$ & $1966: 03$ & \\
Trough & & $1967: 07$ & $1967: 03$ & \\
Peak & & & $1967: 10$ & $1966: 01$ \\
Trough & & & $1968: 11$ & $1966: 09$ \\
Peak & & $1970: 01$ & $1969: 11$ & $1968: 11$ \\
\hline
\end{tabular}

*Peaks and troughs for the US come from Pagan and Sossounov (2002). 
Table 3b. Dates of the Peaks and Troughs in the 1970-1999 period

\begin{tabular}{lccccc}
\hline & SPAIN & FRANCE & ITALY & GERMANY & US \\
\hline Trough & & & & & $1970: 06$ \\
Peak & & $1970: 01$ & & & $1971: 04$ \\
Trough & & $1971: 010$ & $1972: 03$ & $1971: 10$ & $1971: 11$ \\
Peak & $1974: 04$ & $1973: 05$ & $1973: 06$ & $1973: 03$ & $1972: 12$ \\
Trough & & $1974: 09$ & & $1974: 09$ & $1974: 09$ \\
Peak & & $1975: 01$ & & $1976: 03$ & $1976: 12$ \\
Trough & $1978: 02$ & $1977: 04$ & $1977: 12$ & $1976: 10$ & $1978: 02$ \\
Peak & $1978: 07$ & & & $1978: 09$ & \\
Trough & $1979: 11$ & & & $1980: 03$ & \\
Peak & $1981: 08$ & $1980: 10$ & $1981: 05$ & $1981: 07$ & $1980: 11$ \\
Trough & $1982: 09$ & $1981: 06$ & $1982: 07$ & $1981: 12$ & $1982: 07$ \\
$\quad$ Peak & & $1983: 12$ & $1983: 03$ & & $1983: 06$ \\
Trough & & $1985: 10$ & $1983: 12$ & & $1984: 04$ \\
$\quad$ Peak & $1987: 08$ & $1987: 09$ & $1986: 05$ & $1986: 04$ & $1987: 08$ \\
Trough & $1987: 11$ & $1988: 01$ & $1988: 02$ & $1988: 01$ & $1987: 11$ \\
$\quad$ Peak & $1989: 09$ & $1990: 05$ & $1990: 06$ & $1990: 07$ & $1990: 05$ \\
Trough & $1990: 09$ & $1990: 09$ & & & $1990: 10$ \\
$\quad$ Peak & $1991: 05$ & & & & \\
Trough & $1992: 09$ & & $1992: 09$ & $1992: 09$ & \\
Peak & $1994: 01$ & $1994: 01$ & $1994: 05$ & $1994: 04$ & $1994: 01$ \\
Trough & $1995: 03$ & $1995: 02$ & $1995: 12$ & $1995: 03$ & $1994: 06$ \\
Peak & $1998: 07$ & & $1998: 07$ & $1998: 06$ & \\
Trough & $1998: 09$ & & $1998: 10$ & $1998: 09$ & \\
\hline
\end{tabular}

*Peaks and troughs for the US come from Pagan and Sossounov (2002). 


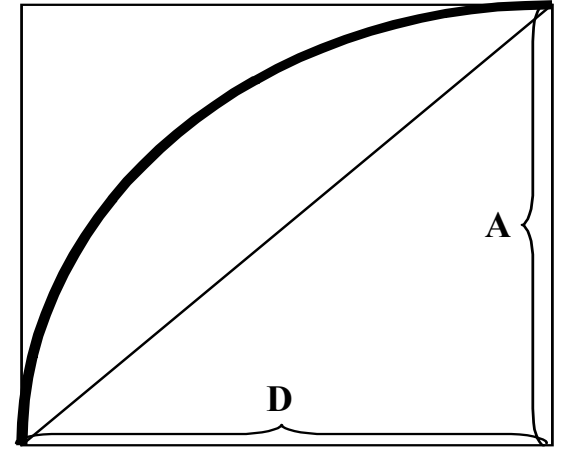

A) A Bull Market with EX $>0$

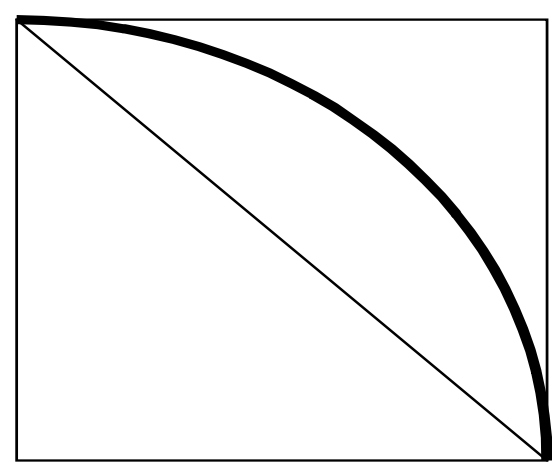

C) A Bear Market with EX $>0$

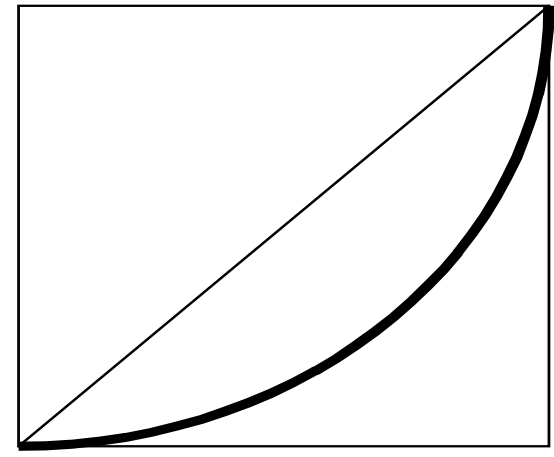

B) A Bull Market with EX $<0$

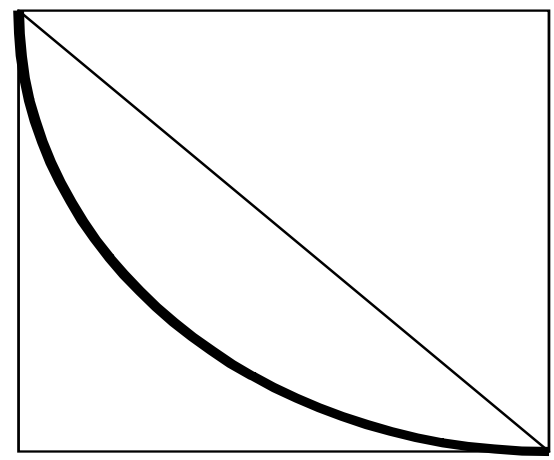

D) A Bear Market with $\mathrm{EX}<0$

Figure 1: Shape of the Bull / Bear Phases and value of the Excess measure 


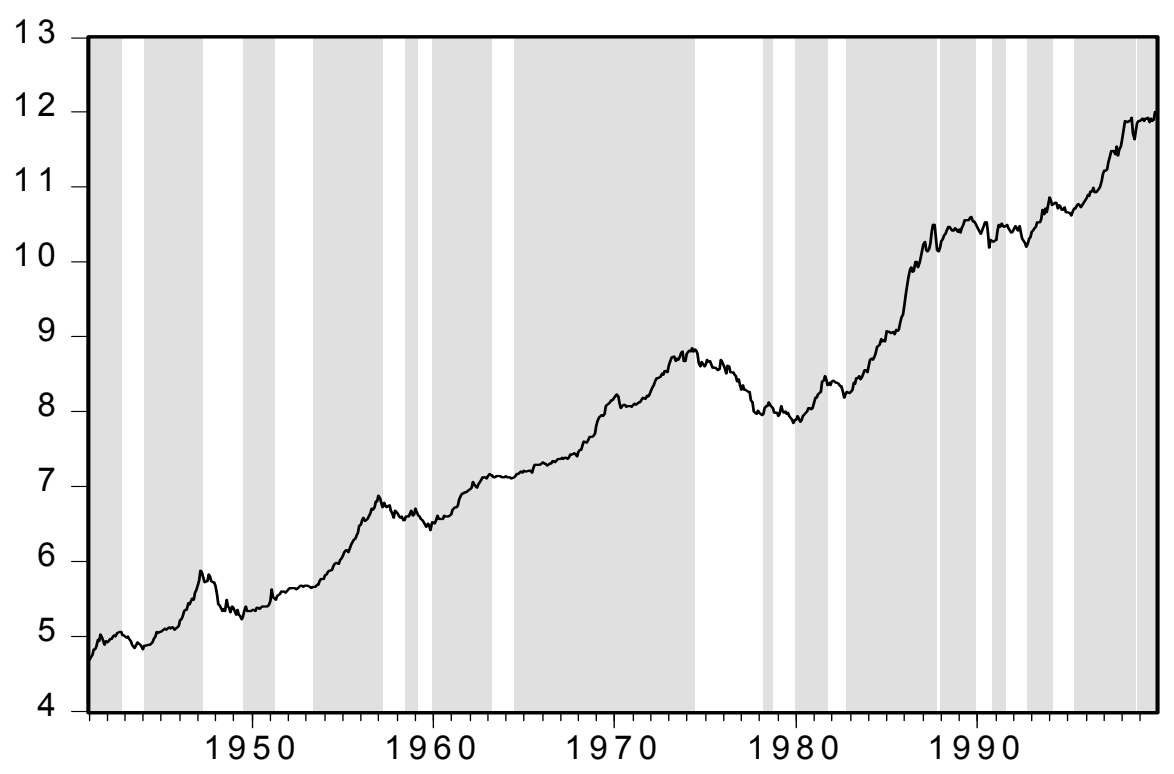

Figure 2: Spanish Stock Index. Bullish Phases Shaded

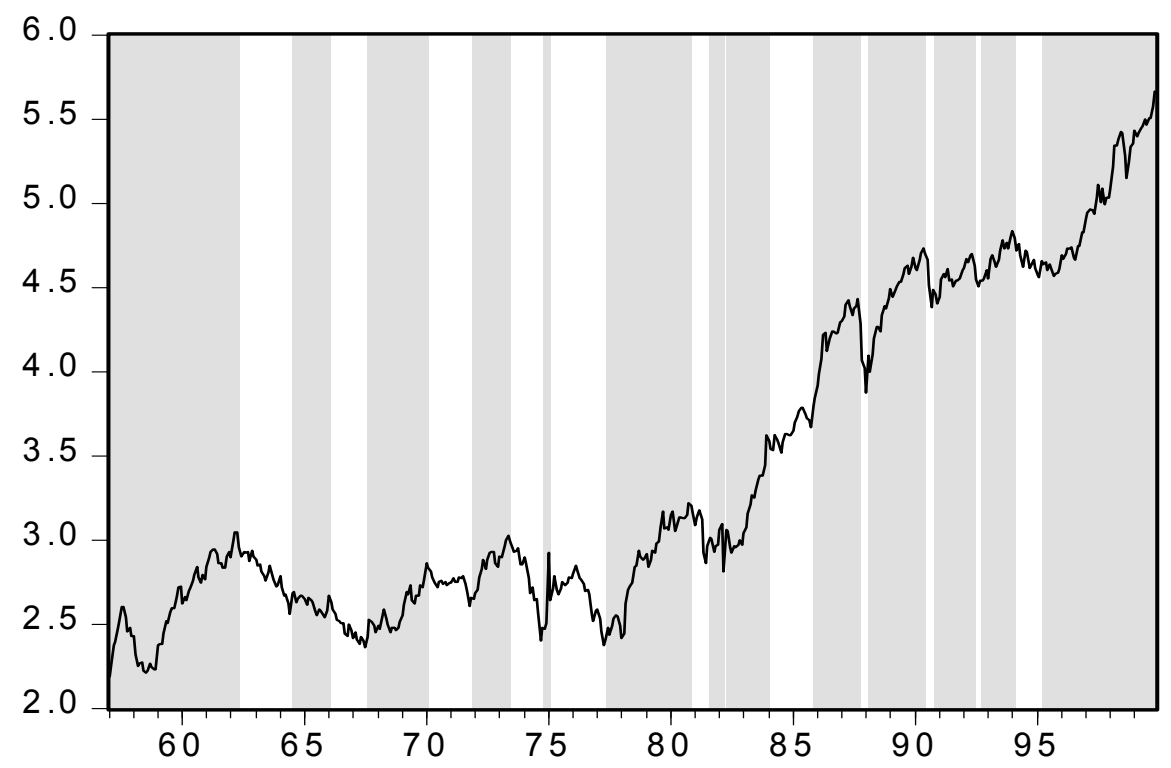

Figure 3: French Stock Index. Bullish Phases Shaded 


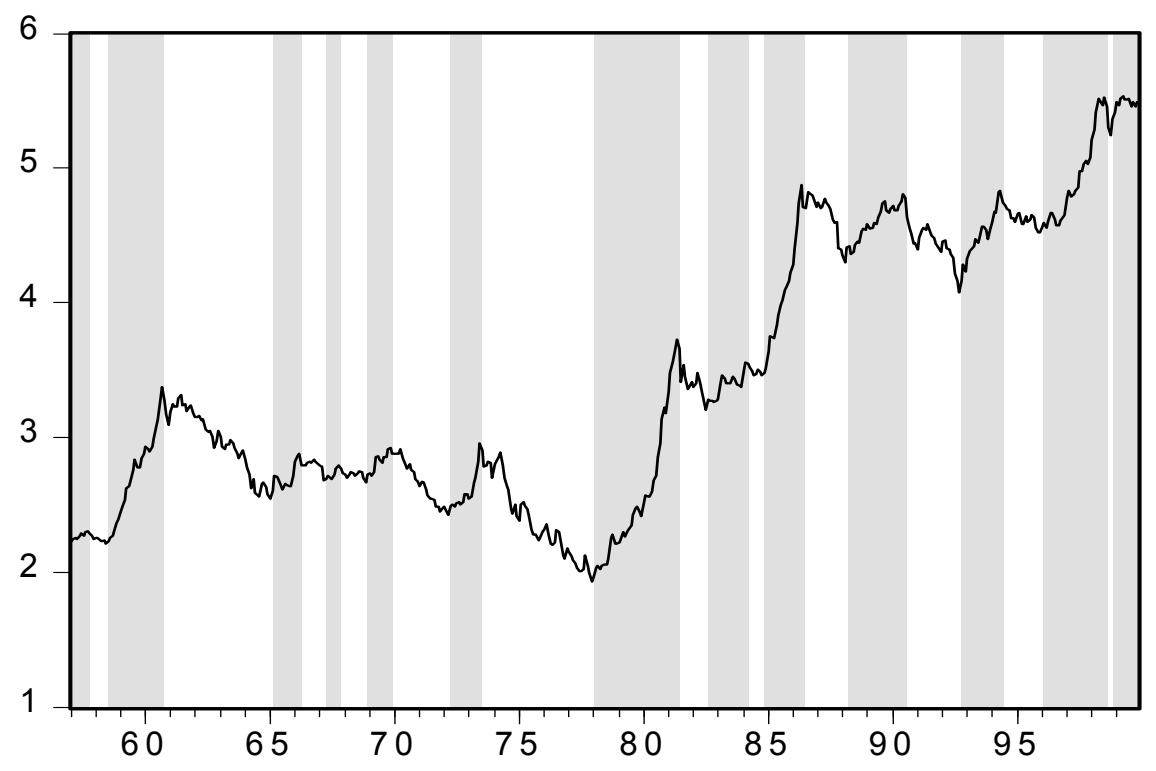

Figure 4: Italian Stock Index. Bullish Phases Shaded

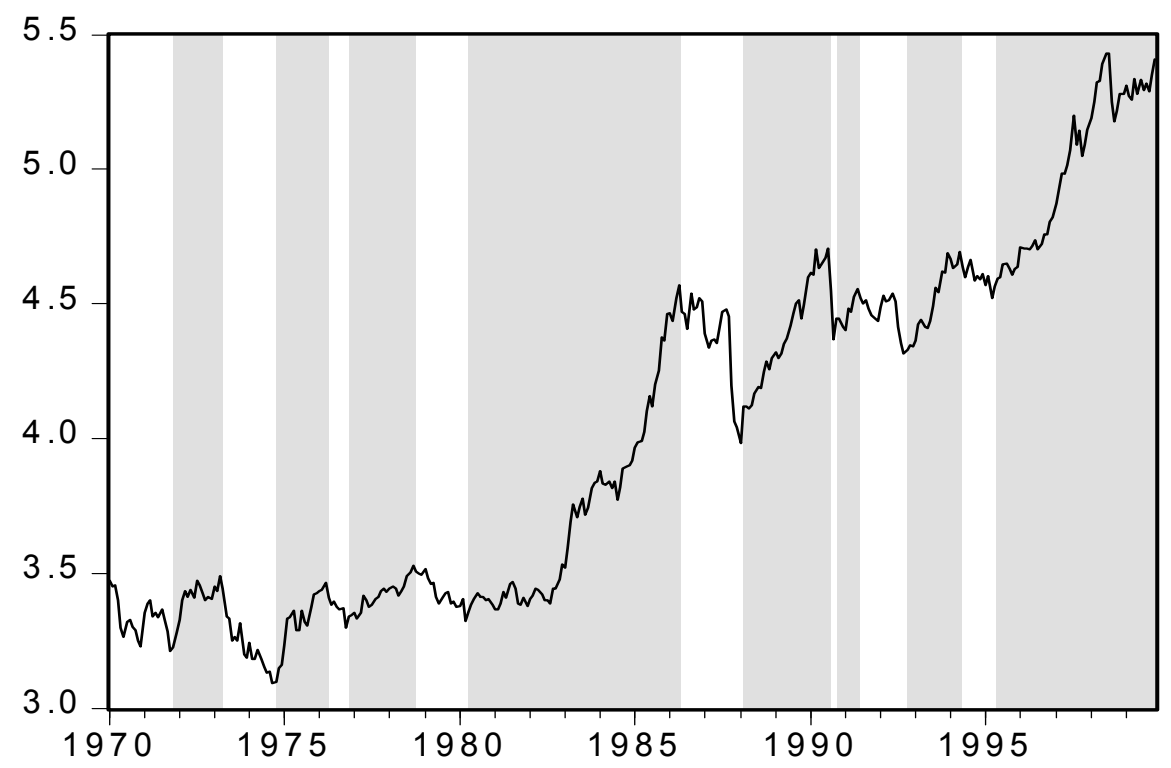

Figure 5: German Stock Index. Bullish Phases Shaded 


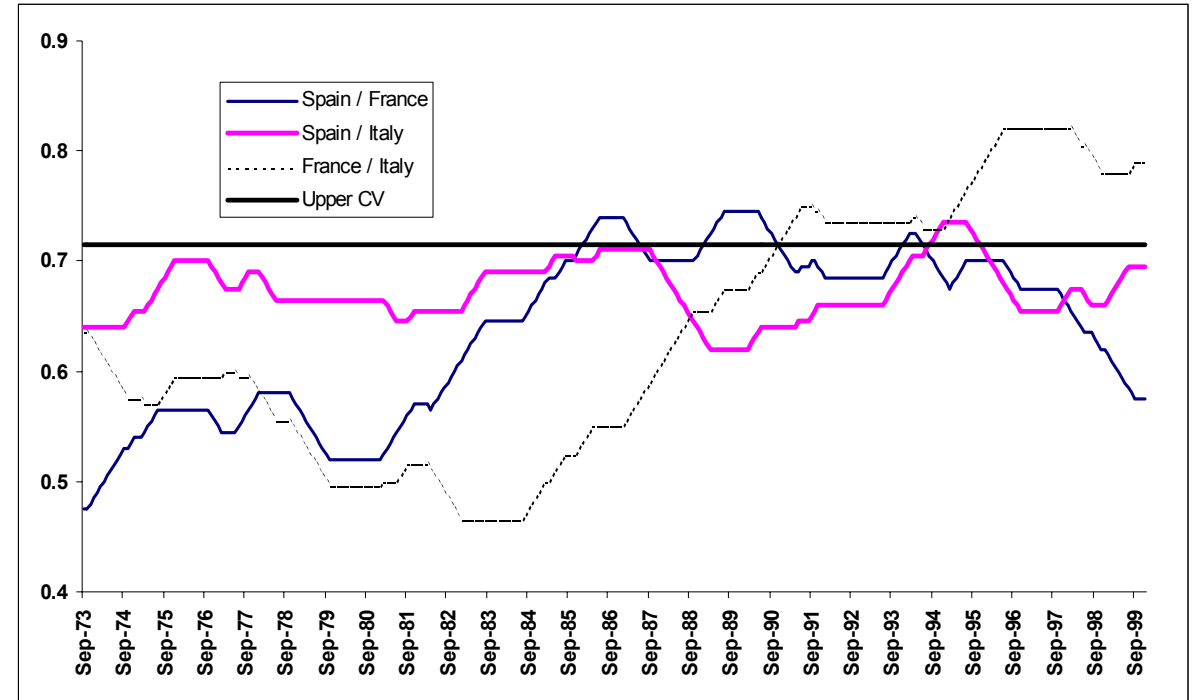

Figure 6: Rolling Coincident Indexes for Spain, France and Italy. The indexes have been calculated using a rolling window of 200 observations. Upper and lower critical values correspond to the $5 \%$ confidence interval of the simulated distribution of the indexes. 


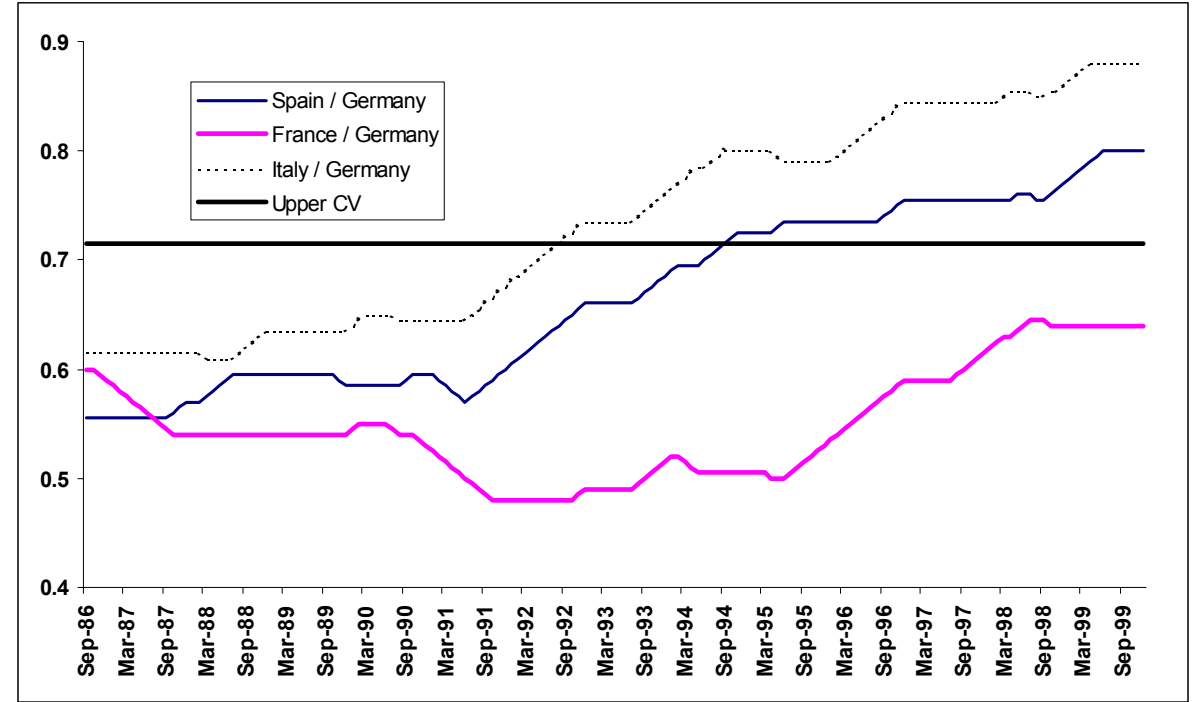

Figure 7: Rolling Coincident Indexes for Germany. The indexes have been calculated using a rolling window of 200 observations. Upper and lower critical values correspond to the $5 \%$ confidence interval of the simulated distribution of the indexes. 